www.jmscr.igmpublication.org

Index Copernicus Value: 79.54

ISSN (e)-2347-176x ISSN (p) 2455-0450

crossrefDOI: https://dx.doi.org/10.18535/jmscr/v7i1.169

\title{
Neurocysticercosis - Clinical and Radiological Appraisal from a Tertiary Centre of Bihar, India
}

\author{
Authors \\ Anju Kumari ${ }^{1}$, Ravikant Kumar ${ }^{2}$ \\ ${ }^{1}$ Lady Medical Officer, Indira Gandhi Institute of Medical Sciences, Patna -800014 \\ ${ }^{2}$ Assistant Professor, Indira Gandhi Institute of Medical Sciences, Patna -800014 \\ Corresponding Author \\ Dr Ravikant Kumar \\ Assistant Professor, Department of Gastroenterology, IGIMS, Patna, India \\ Mobile no.91-7903043715, Email: drravi0609@gmail.com
}

\begin{abstract}
Little has been published on Neurocysticercosis (NCC) in the State of Bihar, India. This study was conducted to describe the clinical and radiological features of NCC.

Materials: Fifty patients of NCC presenting in department of medicine, PMCH, Patna were evaluated on preset proforma containing detailed demographic data, dietary habits, clinical features and neuroimaging findings.

Results: Out of 50 patients of NCC, 28 (56\%) were male with most common age group being 21-30 years (36\%). Seizure only is the most common presentation followed by seizure with increased intracranial pressure (70 and $14 \%$ respectively). Other presentation are seizure with psychotic symptoms, meningoencephalitis and ICSOL. CSF is inflammatory in 50\% and ELISA is positive in 30 (60\%). Multiple ring enhancing lesions either alone or in combination were seen on computerised tomography scans in 28 $(56 \%)$ cases and parietal lobe involvement is the commonest site.

Conclusions: NCC is common public health problem in Bihar and one of the common cause of seizure. High level of suspicion is of paramount importance for diagnosis so that appropriate treatment can be started.
\end{abstract}

Keywords: Bihar, ELISA, NCC, neuroimaging, seizure.

\section{Introduction}

Cysticercosis, the infection caused by larval stage of tapeworm Taenia solium, is the most common parasitic disease of nervous system in human and single most common cause of acquired epileptic seizure in developing world ${ }^{1}$. Cysticerci have a predilection for migrating to the central nervous system (CNS), eyes and striated muscle. When cysticercosis affects CNS or eye it is called neurocysticercosis (NCC). Its prevalence varies greatly according to the geographical region and is not yet precisely known. This study was carried out to describe the clinical and radiological features of NCC in state of Bihar, India.

\section{Material and Methods}

The present prospective study was conducted in department of medicine, Patna Medical College, 
Patna. Fifty patients were included in this study. The diagnosis of neurocysticercosis (NCC) was made on the basis of following criteria-

1) CT finding of single/multiple, low/high attenuating lesion, disc or ring enhancing lesion with compressed ventricle

2) ELISA test for cysticercosis antibody in serum.

3) CSF examination

All patients of NCC were evaluated clinically on preset proforma and subjected to routine haematological and biochemical investigations. CSF examination for biochemical and cytology with particular reference to eosinophil was done. Plain and contrast cranial CT was done in all cases. ELISA for cysticercus antibody was done.

\section{Observations}

Out of 50 patients of NCC, $28(56 \%)$ were male with most common age group being 21-30 years $(36 \%)$. Seizure only is the most common presentation followed by seizure with increased intracranial pressure (70 and 14\% respectively). Other presentation are seizure with psychotic symptoms, meningoencephalitis and ICSOL (table 1). Hyperreflexia $(8,16 \%)$, papilloedema $(5,10 \%)$, disorientation, nuchal rigidity are common signs. Most common seizure pattern were partial seizure with secondary generalisation $(25,55.5 \%)$ followed by generalized tonic clonic seizure (table 2). Table 3 shows haematological and CSF findings. ELISA for NCC is positive in $30(60 \%)$ patients and CSF is inflammatory in half of cases. Fifteen (30\%) presented with ring enhancing lesion alone and 13(26\%) patients with ring enhancing lesion in combination, together they constituted maximum number of cases. Equally common was pin head hyper attenuated lesion found in $8(16 \%)$ and in combination in 19(38\%) patients. Most common site of lesion was parietal lobe (table 4)
Table 1: Sign and symptoms of NCC

\begin{tabular}{|l|c|c|}
\hline Symptoms & Number & Percent \\
\hline Epilepsy & 45 & 90 \\
\hline Headache & 12 & 24 \\
\hline Nausea \pm vomiting & 10 & 20 \\
\hline Altered sensorium & 04 & 08 \\
\hline Psychiatric features & 04 & 08 \\
\hline Visual disturbance & 05 & 10 \\
\hline fever & 03 & 06 \\
\hline Sign & & \\
\hline Nuchal rigidity & 3 & 06 \\
\hline Papilloedema & 05 & 10 \\
\hline Hyperreflexia & 08 & 16 \\
\hline Ataxia & 03 & 06 \\
\hline Disorientation & 05 & 10 \\
\hline
\end{tabular}

Table 2: Clinical seizure pattern

\begin{tabular}{|c|c|c|}
\hline Seizure pattern & Number & Percent \\
\hline Generalised tonic-clonic (GTCS) & 18 & 40 \\
\hline Simple partial & 2 & 4.5 \\
\hline $\begin{array}{l}\text { Partial seizure with secondary } \\
\text { generalisation }\end{array}$ & 25 & 55.5 \\
\hline
\end{tabular}

Table 3: Haematological and CSF finding in NCC

\begin{tabular}{|l|c|c|}
\hline Investigation & Number & Percent \\
\hline Blood & & \\
Peripheral leucocytosis & 15 & 30 \\
Peripheral eosinophilia & 10 & 20 \\
\hline CSF & & \\
\hline Inflammatory CSF & 25 & 50 \\
\hline Non inflammatory CSF & 25 & 50 \\
\hline Eosinophil in sediment & 5 & 10 \\
\hline Serum ELISA & & \\
\hline POSITIVE & 30 & 60 \\
\hline NEGATIVE & 20 & 40 \\
\hline
\end{tabular}

Table 4: Site of lesion on CT Scan

\begin{tabular}{|l|c|}
\hline Site of lesion & Number \\
\hline Frontal lobe & 20 \\
\hline Parietal lobe & 40 \\
\hline Temporal lobe & 10 \\
\hline Occipital lobe & 25 \\
\hline Cerebellum & 05 \\
\hline
\end{tabular}

\section{Discussion}

Neurocysticercosis is one of the most serious problem of public health in developing nation. It has varied clinical presentation. In this study, maximum number (36\%) of patients were encountered in age group of 21 to 30 years and approximately $80 \%$ patents belongs to $11-40$ years group. Verma $\mathrm{A}$ and Gaur $\mathrm{KJ}^{2}$ from Uttaranchal reported that majority of cases were in 
the 21-30 age group. Male outnumbered female (M:F:: 1.27:1). According to Arseni and Sarnitca ${ }^{3}$, 37 were males $(59.6 \%)$ and $40.2 \%$ were female.

Seizure was the most common (45pts, 90\%) presentation in our study. Seizure was the lone presentation in $35(70 \%)$ of cases. Incidence of seizure was found in $50-55 \%$ in the reports available from outside India ${ }^{4,5}$ whereas incidence varies from 59-94\% in studies reported from India $^{6,7}$. In this study partial seizure with secondary generalization was present in $25(55.5 \%)$ and is the commonest seizure type. Trelles and Trelles ${ }^{8}$, Sotelo et $\mathrm{al}^{4}$ found partial seizure with secondary generalization to be the commonest clinical presentation. Second most common seizure type is generalized tonic- clonic $(18,40 \%)$. Kuruvilla et $\mathrm{al}^{9}$ reported generalized seizure as a presenting symptoms in $36 \%$ cases. Feature of raised intracranial tension was present in (7 pts, 14\%). Mukherjee et al ${ }^{10}$ in a study of 30 cases reported an incidence of raised intracranial tension in $19 \%$ cases.

Seizure was the most common symptoms followed by headache $(12,24 \%)$, nausea/ vomiting in $10(20 \%)$. Sotelo et al. ${ }^{4}$ observed headache in $43.4 \%$ and nausea/vomiting in $27.2 \%$. Among the signs, hyperreflexia (16\%) and disorientation $(10 \%)$ were the commonest. Sotelo et $\mathrm{al}^{4}$ observed hyperreflexia in $21 \%$ and bilateral papilledema in $28 \%$. Svetlana Agapejev ${ }^{11}$ reported hyperreflexia in $13(59.1 \%)$ and bilateral papilledema in 17 $(73 \%)$ cases. Discrepancies in the incidence of signs and symptoms in our study as compared to others may be due to less number of patients in this study.

Perirpheral blood smear examination revealed leukocytosis in $15(30 \%)$ cases and peripheral eosinophilia in 10 pts $(20 \%)$. Mccormick et $\mathrm{al}^{5}$ reported leukocytosis in $10 \%$ and eosinophilia in $3.1 \%$ cases where as Grisolia ${ }^{12}$ et al reported in 35 and $17.6 \%$ cases respectively. CSF was inflammatory in $50 \%$ cases. Sotelo et al $^{4}$ observed that when CSF is inflammatory $80 \%$ cases show eosinophil. Grisolia et $\mathrm{al}^{12}$ reported eosinophilia in CSF in $20 \%$ cases. CT scan is helpful not only in suspecting the etiology but also in finding the number, localization and extent of lesion. In our study of 50 patients, $15(30 \%)$ presented with ring enhancing lesion alone and $13(26 \%)$ patients with ring enhancing lesion in combination, together they constituted maximum number of cases. Equally common was pin head hyper attenuated lesion found in 8 patients $(16 \%)$ and in combination in 19 patients $(38 \%)$. Bhatia ${ }^{13}$ in a series of 20 patients of NCC found 7 cases with ring enhancing lesion alone and 6 patients in combination. Majority of ring lesion either isolated or in combination measured $5-10 \mathrm{~mm}$ whereas pinhead and calcified lesion lesion were $<5 \mathrm{~mm}$ in size. Bhatia reported similar finding regarding size of lesions. In our series, parietal lobe involvement was the commonest site (90\%) whereas frontal lobe was affected in $25(50 \%)$. Majority of patients had also shown perifocal edema. Extensive edema frequently seen with NCC helps to distinguish it from cerebritis, small abscesses or metastasis.

\section{Conclusion}

Neurocysticercosis is one of the most serious public health problems and it has varied clinical presentation, seizure being the most common. Features of raised intracranial tension, psychotic behavior, meningoencephalitis and intracranial space occupying lesion are other presentation. Most commonly affected age group is 11-40 years. Peripheral leucocytosis and eosinophilia are found in small number of patients. Parietal site is most common site of involvement and ring enhancing lesion is the commonest CT finding. High level of suspicion is of paramount importance for diagnosis so that appropriate treatment can be started.

\section{Conflict of Interest: None \\ Source of Funding: None \\ Ethical Clearance: Taken}




\section{References}

1. Del Brutto $\mathrm{OH}$, Sotelo J, Roman CG, Therapy for neurocysticercosis: a reappraisal. Clin Infect Dis 1993;17:7305.

2. Varma A, Gaur KJ. The Journal of the Association of Physicians of India 2002, 50:1398-1400

3. Arseni C, Samitica DC. Cysticercosis of brain. Br Med J. 1957 Aug 31; 2(5043): 494-497

4. Sotelo J, Guerrero V, Rubio F. Neurocysticercosis: a new classification based on active and inactive forms: a study of 753 cases. Archives of Internal Medicine 1985;145:442-445

5. McCormick GF,Zee CS, Heiden J. Cysticercosis Cerebri Review of 127 Cases. JAMA Neurology1982; 39(9):534539.

6. Ahuja GK, Roy S, Kamja J, Virmani V. Cerebral cysticercosis. J Neural Sci.1978; 35:365

7. Meena Gupta. Neurocysticercosis: Proceedings of Neurology Update. GB Pant Hospital New Delhi, 1989:34-54.

8. Trelles J.O.; Trelles L. Cysticercosis of the nervous system. in: Handbook of Clinical Neurology 1978; 35:291-320.

9. A Kuruvilla, J D Pandian, M Nair, V V Radhakrishnan, S Joseph. Neurocysticercosis: A Clinical and Radiological Appraisal from Kerala State, South India. Singapore Med J 2001 Vol 42(7) : 297303.

10. Mukherjee A, Roy T, Mukherjee S, Basu N, Duttamunshi A. Neurocysticercosis. JAPI 1993;41: 287-289.

11. Svetlana Agapejev, Maria Dorvalina da Silva, Anete K. Ued. Severe forms of neurocysticercosis- Treatment with albendazole. Arq Neuropsiquiatr 1996;54(1): 82-93
12. Grisolia S, Wiederholt W C. CNS Cysticercosis. Arch Neurol. 1982;39(9): 540-544.

13. Dhamija RM, Venkatraman S, Sanchetee PC, Roy AK, Bhatia HC. Computed tomographic spectrum of neurocysticercosis. J Assoc. Physicians India. 1990;38: 566-568. 